\title{
Advanced Electron Microscopy for Materials Science
}

\author{
Zentaro Akase ${ }^{1}$, Mitsuaki Higo ${ }^{1}$, Keiko Shimada ${ }^{2}$, Takafumi Sato ${ }^{1}$, Hideyuki Magara ${ }^{1}$, \\ Daisuke Shindo ${ }^{2, *}$ and Nobuhiko Ohno ${ }^{3,4}$ \\ ${ }^{1}$ Institute of Multidisciplinary Research for Advanced Materials, Tohoku University, Sendai 980-8577, Japan \\ ${ }^{2}$ RIKEN Center for Emergent Matter Science (CEMS), Wako 351-0198, Japan \\ ${ }^{3}$ National Institute for Physiological Sciences, Okazaki 444-8585, Japan \\ ${ }^{4}$ School of Medicine, Jichi Medical University, Shimotsuke 329-0498, Japan
}

\begin{abstract}
This paper presents current research trends in advanced electron microscopy techniques for materials science. The survey is based on the special issue of Materials Transactions published in October 2019 (Vol. 60, No. 10). Advanced electron microscopy has been applied extensively to characterize various materials. The recent development and extension of analyses of electric fields and the collective motions of secondary electrons by in situ electron holography are discussed in detail. [doi:10.2320/matertrans.MT-M2021086]
\end{abstract}

(Received May 14, 2021; Accepted July 12, 2021; Published September 3, 2021)

Keywords: electron holography, dynamical diffraction, in situ experiment, electric field, secondary electron

\section{Introduction}

Transmission electron microscopy (TEM) has been used to clarify the microstructures of various functional materials, and high-resolution $\mathrm{TEM}^{1-5)}$ has been used to directly observe atomic arrangements. Such detailed atomic arrangements projected down along the incident electron beam can be observed with a resolution limit of less than $0.1 \mathrm{~nm}$. By using microprobes smaller than $0.1 \mathrm{~nm}$ and beam scanning systems, scanning transmission electron microscopy (STEM) is used for obtaining atomic-level elemental mapping. ${ }^{6-9)}$ In addition to this improvement in resolution, researchers have developed techniques for analyzing the distribution of the electromagnetic field inside and outside of specimens by focusing on the phase information of the incident electron beam. A typical technique is electron holography, which utilizes the interference of electron waves. ${ }^{10-13)}$

Although numerous review and overview papers, including books, describing the applications of TEM in materials science are available, ${ }^{4,14,15)}$ a special issue was published in October 2019 in Materials Transactions (Vol. 60, No. 10) under the title "Development and Application of Advanced Electron Microscopy Techniques for Materials Science". This special issue contains a total of five articles covering research on the wave behavior of electrons ${ }^{16,17)}$ and analyses of materials and devices using electron holography. ${ }^{18,19)}$ In addition to electron holography, various techniques such as dark-field imaging and high-angle annular dark-field (HAADF) STEM observations have been used to characterize charged-domain walls (CDWs) in ferroelectric

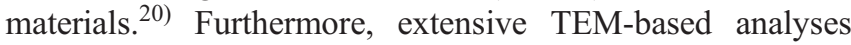
have recently been published. ${ }^{21-23)}$

Before this special issue was published, the 27th Meeting of the Materials Science and Engineering Research Society was held at the Institute of Multidisciplinary Research for Advanced Materials, Tohoku University, with the theme "Recent Trends in Materials Properties and Microscopy Techniques". This special issue was assembled by collecting papers from some of the participants of the meeting. In

*Corresponding author, E-mail: daisuke.shindo@ riken.jp relation to electron holography, review articles are available. ${ }^{13,24)}$ Also, a Microscopy special issue on "Electron Interference Microscopy" has recently been published. ${ }^{25-35)}$

In the following sections, we review the special issue of Materials Transactions (Vol. 60, No. 10), and present the latest research developments of our research group related to the special issue.

\section{Review of the Special Issue}

\subsection{Generation and control of electron vortex beams}

In the special Materials Transactions issue on "Development and Application of Advanced Electron Microscopy Techniques for Materials Science", Harada et al. ${ }^{16)}$ reported generating vortex beams by using fork-shaped gratings manufactured by focused-ion-beam (FIB) instruments to clarify the performance of vortex beams. They generated vortex beams using a small-angle electron diffraction optical system $^{36)}$ and also successfully controlled vortex beams by changing the shapes and sizes of openings. The electron vortex beams can carry angular momenta while propagating $^{37-40)}$ and can potentially be used to detect the magnetic flux perpendicular to the specimen plane owing to its angular momentum, in addition to the in-plane components (perpendicular to the electron beam) of the magnetic flux owing to the Lorenz force. ${ }^{41-43)}$ Furthermore, electron vortex beams can potentially be used for microfabrication and magnetization control. These possible applications indicate that electron vortex beams and other extended electron beams such as Bessel beams are potential next-generation electron beam technologies. ${ }^{44-47)}$

\subsection{Effect of dynamical electron diffraction on electron phase shift}

The aforementioned Materials Transactions special issue includes Akase et al.'s ${ }^{17)}$ investigation of the effect of dynamical electron diffraction on the phase shift ${ }^{48-51)}$ in electron holograms recorded from a wedge-shaped specimen of single-crystal $\mathrm{Si}$ around a Bragg diffraction condition. Their results show that the effective inner potential depends on the direction of the incident electron beam, especially 
near the Bragg condition. The characteristic phase shift has been analyzed using the dynamical theory of electron diffraction. ${ }^{52)}$

Figure 1 shows bright-field images, reconstructed phase images, and phase-shift profiles without (a) and with (b) the 220 Bragg condition. When the specimen does not satisfy the Bragg condition, the phase shift increases in proportion to the specimen thickness, as shown in Fig. 1(a). However, when the specimen satisfies the 220 Bragg condition, as shown in Fig. 1(b), the phase-shift jumps $\pi$ radians at positions where dark thickness fringes appear in the corresponding bright-field image. These $\pi$ radian jumps can be explained by the dynamical theory of electron diffraction, as shown in Fig. 2. Each column in Fig. 2 shows different diffraction conditions. The upper row shows plots of the complex value of $\psi_{0} \exp (-2 \pi i|\chi| t)$ on the complex plane with the thickness $t$ as a parameter, where $\psi_{0}$ is a wavefunction of the object wave and $\chi$ is the wavenumber vector for the incident electrons in vacuum. The amplitude of this complex value corresponds to the square root of the intensity, and the angle of the complex value corresponds to the phase shift. The thickness ranges from 0 to $300 \mathrm{~nm}$. The data corresponding to $20-80 \mathrm{~nm}$ are plotted as red bold lines in Fig. 2. The middle and lower rows show the intensity and phase shift plotted as functions of the thickness $t$, respectively. In the center column of Fig. 2, the specimen is under an exact 220 Bragg diffraction condition, where the trajectory of $\psi_{0} \exp (-2 \pi i|\chi| t)$ intersects the origin of the complex plane. At the thickness of the intersection, the amplitude of the complex value is zero; the corresponding position in the bright-field image therefore appears dark. Notably, the phase of $\psi_{0} \exp (-2 \pi i|\chi| t)$ was turned over by $\pi$
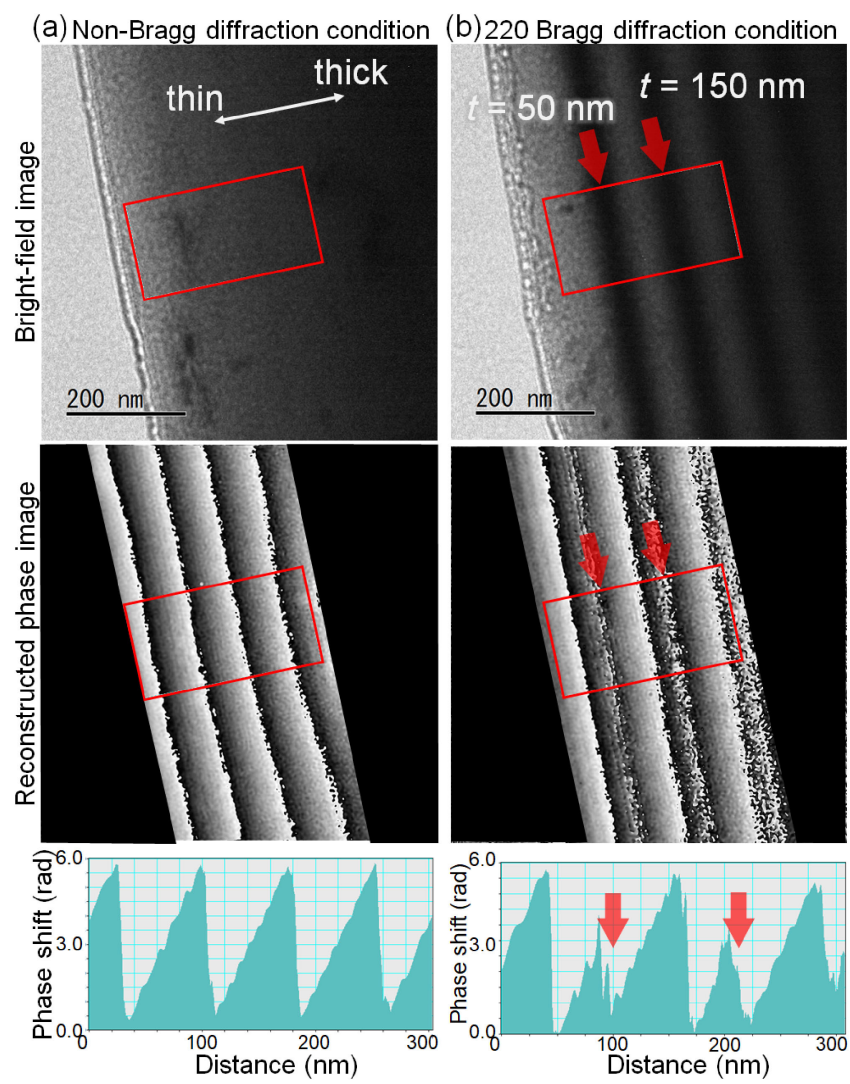

Fig. 1 Bright-field images, reconstructed phase images, and phase-shift profiles for a wedge-shaped Si crystal. ${ }^{17)}$ (a) Strong Bragg reflections are not excited. (b) The Bragg diffraction condition with 220 reflections is exactly satisfied. The red arrows indicate the positions of dark thickness fringes.
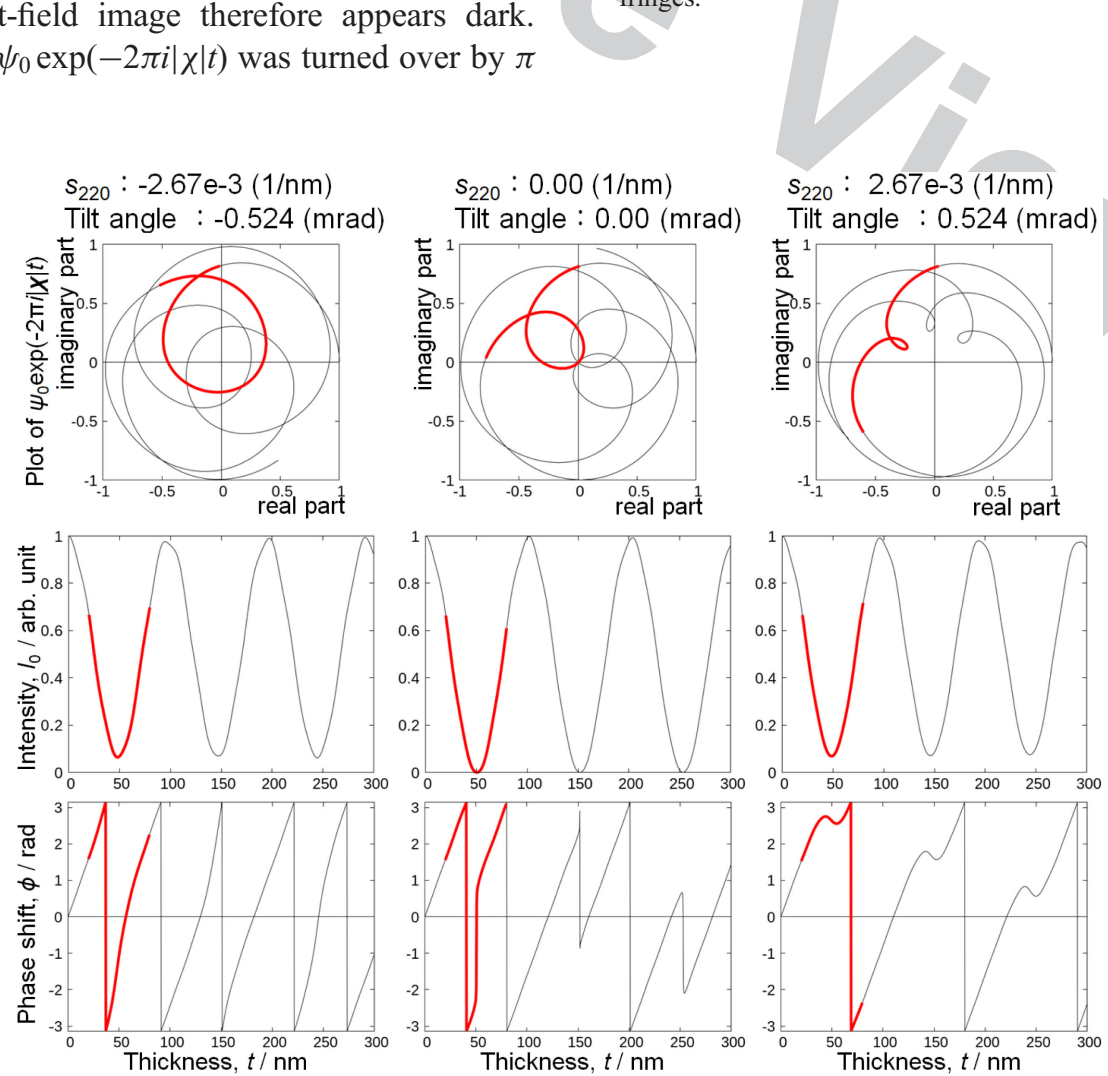

Fig. 2 Multibeam dynamical electron diffraction theory (Bethe method) calculations. ${ }^{17)}$ The columns show different diffraction conditions. The upper row shows the complex value $\psi_{0} \exp (-2 \pi i|\chi| t)$ plotted on a complex plane with thickness as a parameter. The middle and lower rows show the intensity and phase shift plotted as a function of the thickness. The data from $20 \mathrm{~nm}$ to $80 \mathrm{~nm}$ are plotted as red lines. 
across the origin of the complex plane. Therefore, the phase shift profile shows jumps of $\pi$ at the dark thickness fringes. The left and right columns in Fig. 2 show the results when the sign of the excitation error of the 220 reflection is negative and positive, respectively. In these cases, a thickness fringe also appears in the bright-field image; however, the dark fringe intensity does not become zero (see middle row of Fig. 2), and no jumps are observed in the phase-shift profile (see lower row of Fig. 2). Notably, the gradient of the phase shift to the thickness, i.e., the "effective inner potential", depends on the sign of the excitation error of the 220 reflection (see lower row of Fig. 2). This tendency was confirmed by a series of experimentally reconstructed phase images with different incident beam directions.

\subsection{Electric field analysis of precipitates in multilayer ceramic capacitor}

Kawamoto et al. have demonstrated a unique characterization method based on electron holography for determining the electrical properties of submicrometer precipitates produced in multilayer ceramic capacitors (MLCCs). ${ }^{18)}$ They showed that equipotential contour lines present essential information about the electrical conductivity of submicrometer-scale precipitates formed in commercial $\mathrm{BaTiO}_{3}$ MLCCs. Researchers have reported that added $\mathrm{Mg}$ and rare-earth elements play an important role in controlling the temperature dependence of the dielectric constant. ${ }^{53)}$ However, the addition of such elements induces complex precipitation within the dielectric substance (e.g., a Cr-rich phase, ${ }^{54)}$ Si-rich phase, ${ }^{55}$ and others). Because these precipitates have dielectric constants that differ substantially from that for the $\mathrm{BaTiO}_{3}$ matrix, they are likely to degrade the performance of the capacitor. Thus, studying the local electrical properties in the neighborhood of submicrometerscale precipitates is important.

In their study, Kawamoto et al. used electron holography with handling microprobes in $\mathrm{TEM}^{56-58)}$ to characterize submicrometer-scale precipitates formed in $\mathrm{MLCCs}^{59)}$ and identify the objects that cause harmful electric breakdowns. Figure 3(a) shows a schematic of a thin-foil specimen $(20 \mu \mathrm{m} \times 6 \mu \mathrm{m} \times 100 \mathrm{~nm})$ with a stacked configuration comprising a Ni cathode, dielectric substance $\left(\mathrm{BaTiO}_{3}\right)$, and a $\mathrm{Ni}$ anode. Note that the cathode and anode are physically connected to Pt prongs, which are contacted by metallic PtIr probes when an electrical voltage is applied to the specimen. Figure 3(b) shows an ADF-STEM image of the specimen. A precipitate appearing with dark contrast is indicated by a green arrow. Energy-dispersive X-ray spectroscopy (EDS) analysis shows that this precipitate is rich in $\mathrm{Cr}$ and poor in Si. Figure 3(c) is a reconstructed phase image under an effective applied voltage of $7 \mathrm{~V}$; thus, the authors subtracted the phase-shift information acquired under an applied voltage of $3 \mathrm{~V}$ from that acquired under $10 \mathrm{~V}$ to suppress unwanted phase information such as substantial changes in the mean inner potential at the positions of the precipitates, thickness variations in the specimen, and the magnetic field from the Ni electrodes. Notably, a change occurred in both the spacing and directions of the contour lines in the local area of the precipitate, which was attributed to the difference in the electrical conductivity between the precipitate and the
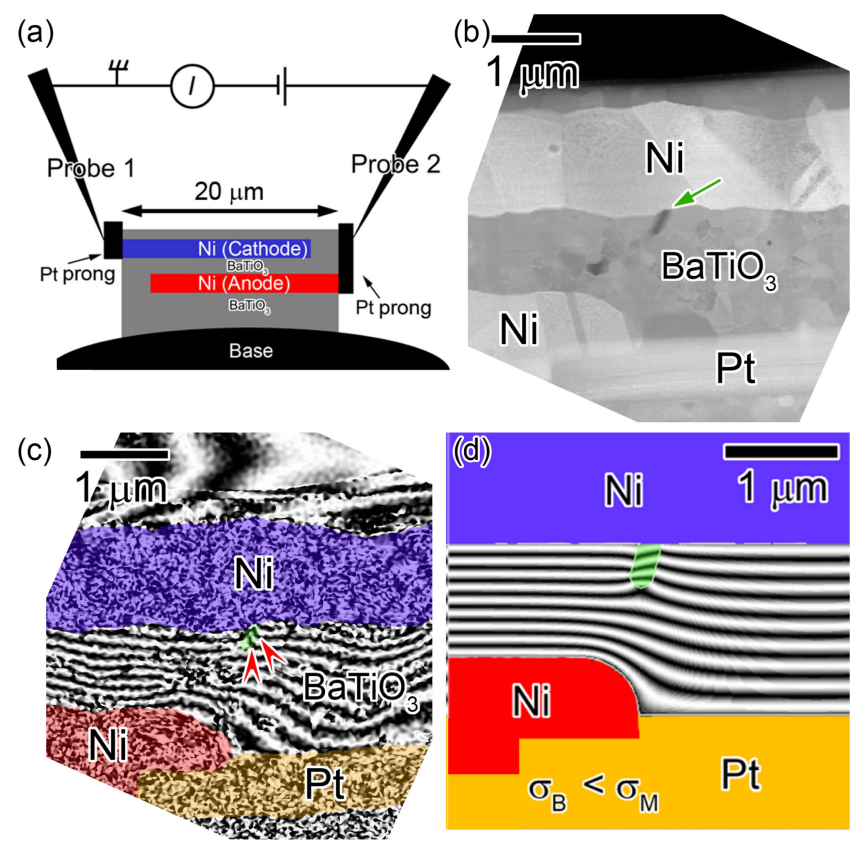

Fig. 3 (a) Schematic of a thin-foil specimen of a multilayer ceramic capacitor. (b) ADF-STEM and (c) reconstructed phase images around a precipitate indicated by an arrow and arrowheads. The phase information is amplified by a factor of two in the reconstructed phase image in (c). (d) Electric-field distribution images obtained by a simulation for the relationship $\sigma_{\mathrm{B}}<\sigma_{\mathrm{M}}$ between the electrical conductivities of the precipitate and the $\mathrm{BaTiO}_{3}$ matrix. ${ }^{18)}$

$\mathrm{BaTiO}_{3}$ matrix. Figure 3(d) shows a two-dimensional simulated electric field map based on finite element method (FEM) modeling. In this model, the electrical conductivity of the precipitate $\sigma_{\mathrm{B}}$ is substantially smaller than the conductivity of the matrix $\sigma_{\mathrm{M}}$. The results in Fig. 3(d) explain several features of the observations (Fig. 3(c)), such as the reduced spacing of the contour lines within the precipitate and the gradual change in the spacing in the matrix area. The conductivity of the Cr-rich phase should be smaller than that of the matrix by two or three orders of magnitude. This method can lead to a deeper understanding of the relationship between the complex microstructure and the material functionalities in capacitors widely used in industry.

\subsection{Characterization of charged-domain walls in ferro- electric material}

Nakajima et al. ${ }^{20}$ examined CDW structures $^{60)}$ in $\mathrm{Ca}_{3-x} \mathrm{Sr}_{x} \mathrm{Ti}_{2} \mathrm{O}_{7}$ (CSTO) using TEM. Ferroelectricity in CSTO has been observed both by conventional electric polarization measurements and by piezoresponse force microscopy. ${ }^{61)}$ The latter revealed two types of CDWs in CSTO in terms of polarity and conductivity: high-conductivity "head-to-head" walls and low-conductivity "tail-to-tail" walls. CDWs are promising for device applications because their morphology and conductivity can be controlled by electric fields and other physical phenomena. ${ }^{62-67)}$ Nakajima et al. ${ }^{20)}$ used dark-field imaging, which is based on the breakdown of Friedel's law, to determine the microstructural characteristics of CSTO. HAADF-STEM observations have demonstrated irregularity of the stacking in the perovskite-structured blocks. Furthermore, electron holography has revealed regions of irregular stacking that produce changes in the mean inner 
potential because of the Sr content. These microscopy studies improve our understanding of the mechanisms by which ferroelectric domains develop in CSTO.

\subsection{Analysis of secondary electrons around a charged epoxy film}

We have thus far described various TEM techniques for materials science, including methods for observing electric fields by electron holography. As an extension of these studies, one of the most interesting subjects is visualizing the motion of electrons because electromagnetic fields basically originate from these motions. The earliest of these studies focused on the charging effect on biological specimens. ${ }^{68,69)}$ The accumulation and distribution of electrons were subsequently observed for various insulating materials, with utmost attention devoted to their surfaces.

Sato et al. ${ }^{19)}$ studied the distribution of secondary electrons around a charged epoxy film prepared by ultramicrotomy. Figure 4(a) shows a reconstructed phase image of a thin film of epoxy resin (dark-brown region). The electric potential of the specimen's surface was estimated to be $1.2 \mathrm{~V}$ by comparison with a simulated reconstructed image. Although no metallic elements were present on the surface of the specimen, the charging effect was considered to be suppressed by irradiation by secondary electrons from the specimen support plate near the observed area. The reconstructed amplitude image in Fig. 4(b) shows red regions around the surface of the epoxy resin, which are considered to correspond to a high density of secondary electrons strongly interacting with the surface of the positively charged specimen. In particular, in the concave region indicated by the arrow in Fig. 4(b), a bright red region is evident; this region corresponds to a large electric field fluctuation due to interaction of the accumulated secondary electrons with the surface. After a hologram of the epoxy resin was observed,
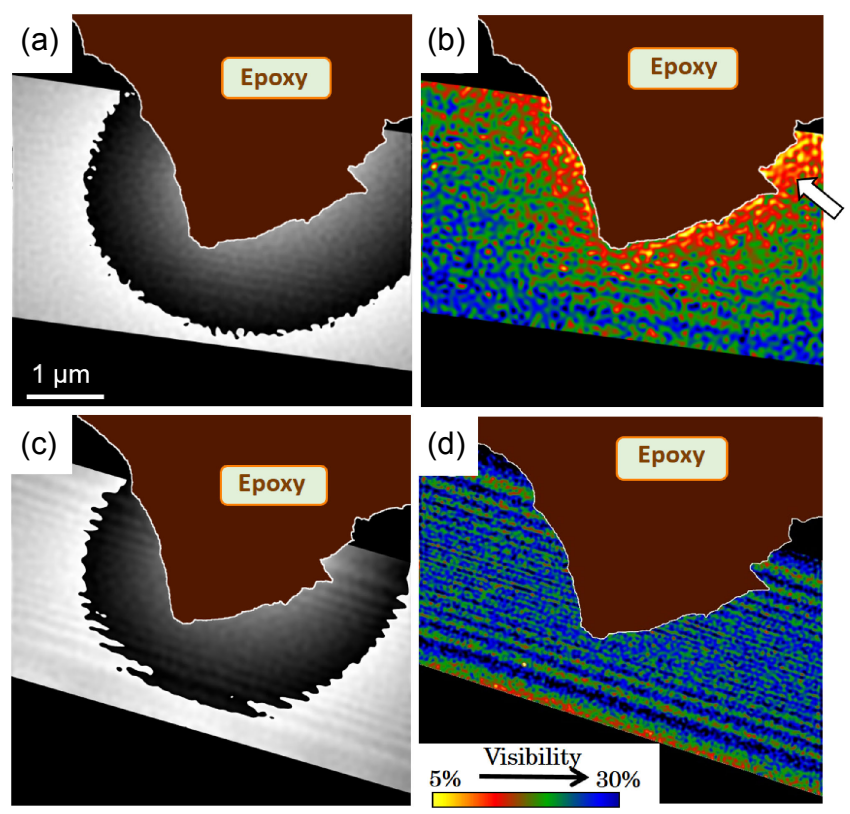

Fig. 4 (a) Reconstructed phase image of ultramicrotomed epoxy resin. (b) Reconstructed amplitude image of resin. (c) Reconstructed phase image of FIB processed resin. (d) Reconstructed amplitude image of FIB processed resin. ${ }^{19)}$ both sides of the thin specimen were irradiated with an FIB system using a weak $\mathrm{Ga}^{+}$-ion beam. The beam intensity was $0.85 \times 10^{3} \mathrm{mC} \cdot \mathrm{m}^{-2}$, which is 200 times smaller than that typically used for polishing specimens. The reconstructed phase image in Fig. 4(c) shows that the electric potential of the specimen becomes $1.0 \mathrm{~V}$ according to the simulation results. The electric potential of the specimen before and after irradiation did not substantially differ because a weak $\mathrm{Ga}^{+}$ion beam was used. In the reconstructed amplitude image in Fig. 4(d), the colored regions observed in Fig. 4(b) are no longer visible. Thus, the distribution of secondary electrons that interact strongly with the surface of the positively charged epoxy resin is sensitive to the presence of metallic elements on the surface.

\section{Extension of In Situ Studies on Collective Motion of Secondary Electrons}

On the basis of these studies on the distribution of secondary electrons on the charged insulating films, in situ experiments can be conducted to investigate the interaction of insulating specimens. Figure 5 shows the results of an in situ study of the electric field variations and the change in secondary electron distribution between two insulating materials: a square pillar of epoxy resin prepared by the FIB method with $\mathrm{Ga}^{+}$ions, and a flake of kidney tissue embedded in epoxy resin and prepared by microtomy. ${ }^{70)}$ The geometric configurations of the square pillar and kidney flake are shown in Fig. 5(c). The square pillar of epoxy resin set in the piezodriving probe of a TEM specimen holder ${ }^{56)}$ approached the kidney flake gradually. In the reconstructed phase images of Fig. 5(a), because of mutual irradiation with secondary electrons, the electric potential of both specimens decreases as their separation decreases. In the reconstructed amplitude images in Fig. 5(b), red- and yellow-colored regions are observed around the kidney flake but not around the square pillar, which is consistent with the observations presented in Fig. 4. When the separation of the specimens fell below approximately $1 \mu \mathrm{m}$, most of the red- and yellow-colored regions disappeared, indicating a decrease in the number of secondary electrons around the kidney flake. Nonetheless, faint color regions remain in the lower-right region, some distance from the pillar of epoxy resin. The visibilities of the interference fringes in the two green " $\mathrm{f}$ " and yellow "e" square regions indicated in the leftmost image of Fig. 5(a) are compared as functions of the separation between the specimens, as shown in Fig. 5(d), as the pillar moves toward $(\bullet)$ or away from $(\boldsymbol{\Delta})$ the kidney flake. A prominent increase in visibility is observed in the yellow square near the kidney flake when the square pillar of epoxy resin approaches the kidney flake surface. The decrease in the number of secondary electrons on the surface of the kidney flake results from the presence of a conductive layer on the surface of the epoxy resin, through which secondary electrons were transferred.

As noted, the accumulation and collective motions of electrons around various positively charged insulators have been observed through the amplitude reconstruction process in electron holography. Those experiments were performed by TEM with a thin insulating specimen surface. Here, we 

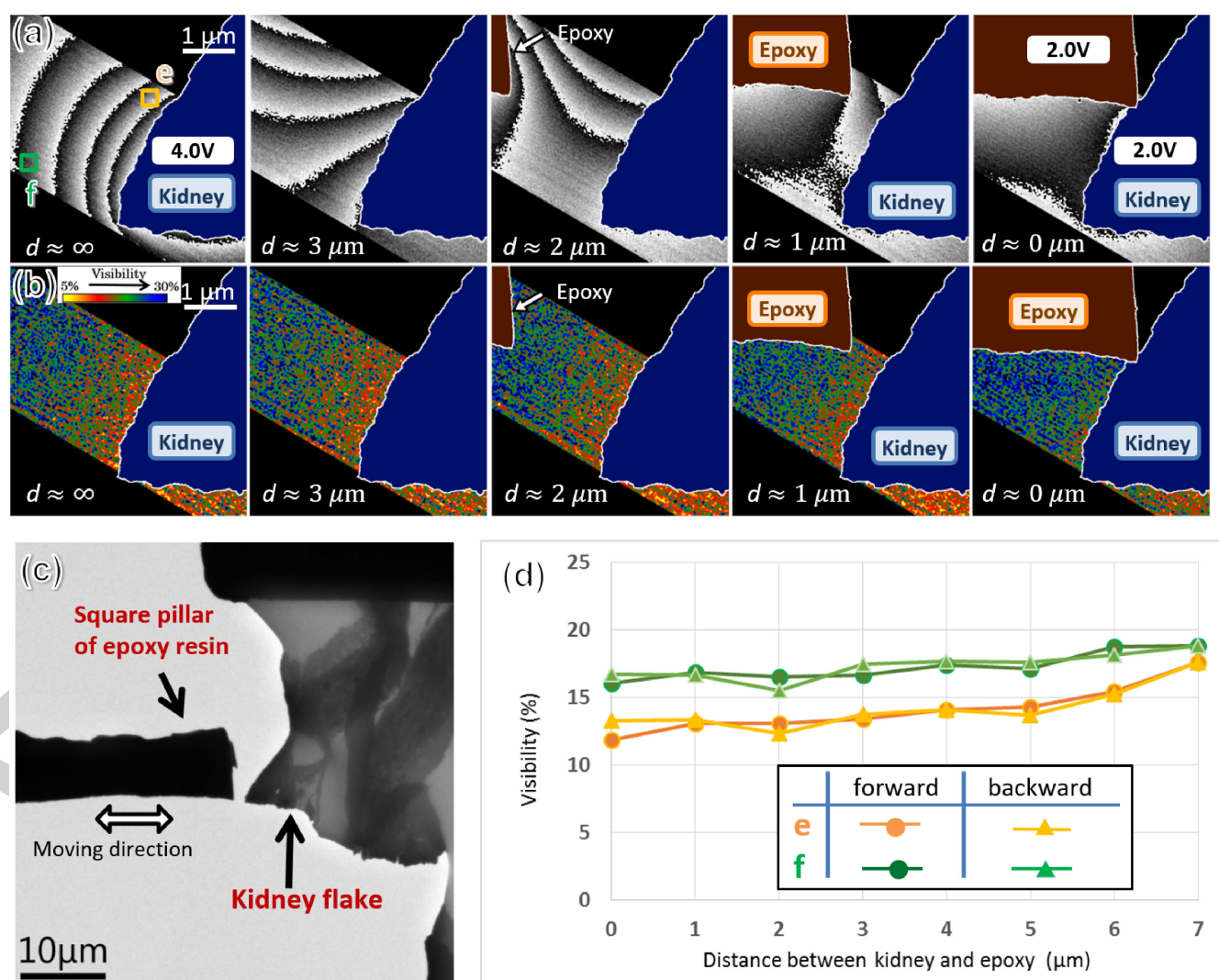

(d)

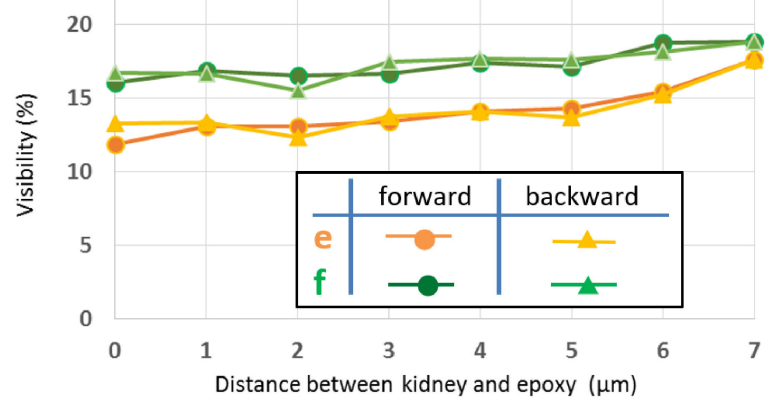

Fig. 5 Secondary electron distributions as a function of separation of epoxy resin and kidney flake. (a) A series of reconstructed phase images in a scenario where a square pillar of epoxy resin is moved toward a kidney flake. The distance $d$ between two insulating materials is indicated in the lower-left part of each figure. (b) Corresponding reconstructed amplitude images. (c) Geometrical configuration of the square pillar of epoxy resin and the kidney flake. (d) Visibility in the square regions denoted by "e" and "f" in the left figure of (a), plotted as a function of the distance between the kidney and the epoxy resin pillar.
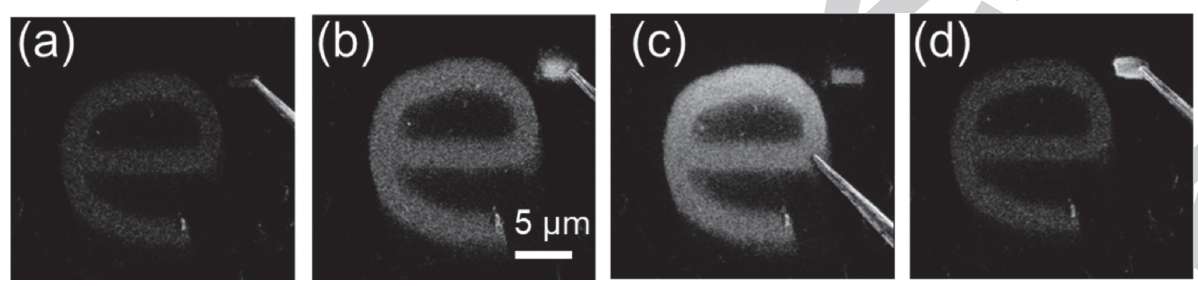

Fig. 6 Successively recorded SIM images of " $\mathrm{e}^{-}$" characters prepared on a mica substrate by W deposition. (a) W islands not touched by +5 V-biased probe needle. (b) The "-" character touched by the probe. (c) The "e" character touched by the probe. (d) The "-" character touched by the probe with a $0.17 \mathrm{ion} / \mathrm{nm}^{2}$ additional $\mathrm{Ga}^{+}$dose after scene (b).

show that similar effects of secondary electrons, which are attracted even to bulk surfaces of insulators, can be observed by scanning ion microscopy (SIM) with an FIB system. The common feature of the phenomena observed by TEM and SIM is the positive charging effect of insulators. In the latter case, during SIM observations, a $\mathrm{Ga}^{+}$-ion probe is used to scan the bulk insulator surface. At each point in the scanned area, secondary electrons are emitted from the bulk surface, whereas $\mathrm{Ga}^{+}$ions accumulate in the bulk specimen, resulting in positive charging. Figure 6 shows a bulk specimen in which $\mathrm{W}$ islands were deposited on a mica substrate to form the character "e" and a superscript minus symbol, "-". Figure 6(a) corresponds to the initial state where W islands are not touched by $+5 \mathrm{~V}$-biased probe needle. When the probe touched one of the islands, "-", the other island, "e", also became brighter (Fig. 6(b)), implying the charge transfer from the island "-" to the island "e" with the existence of metallic elements $\mathrm{Ga}$ and $\mathrm{W}$ on the mica substrate like the case of epoxy thin film in Fig. 4. The existence of $\mathrm{W}$ results from its spread during the deposition process. When the probe directly contacted the character "e", it became much brighter (Fig. 6(c)). On the other hand, in Fig. 6(d), the character "-" again became brighter when touched by the probe but the "e" did not become bright. The SIM observation was continued for $48 \mathrm{~s}$ from scene (b) to scene (d), and the nominal $\mathrm{Ga}^{+}$dose during this period was $0.027\left(\mathrm{C} \mathrm{m}^{-2}\right)$ or 0.17 (ion $\left.\mathrm{nm}^{-2}\right)$. In Fig. 6(d), it is considered that remarkable sputtering of specimen surface by $\mathrm{Ga}^{+}$ions results in the different surface condition from those of Fig. 6(b) and (c). 


\section{Summary}

Research activity related to microstructure and electromagnetic field analyses using TEM continues to increase. The new trends in these microstructure and electromagnetic field analyses were discussed at the 27th Meeting of the Materials Science and Engineering Research Society, held at the Institute of Multidisciplinary Research for Advanced Materials, Tohoku University, with the theme of "Recent Trends in Materials Properties and Microscopy Techniques". On the basis of this meeting, some recent trends related to TEM analyses were published in October 2019 in a special issue of Materials Transactions (Vol. 60, No. 10) under the title "Development and Application of Advanced Electron Microscopy Techniques for Materials Science". As an extension of electron holography on secondary electrons around charged insulating specimens, the interaction between such specimens has been clarified through in situ experiments. In addition, similar effects of secondary electrons, which are attracted to the bulk surface of insulators, have been also observed by SIM with an FIB system. These in situ experiments on secondary electron distributions are expected to be carried out widely and analyzed quantitatively in the near future.

\section{REFERENCES}

1) J.M. Cowley: Diffraction Physics, (North-Holland, New York, 1990).

2) S. Horiuchi: Fundamentals of HREM, (North-Holland, Amsterdam, 1994).

3) J.C.H. Spence: Experimental High Resolution Electron Microscopy, (Oxford University Press, New York, 1988).

4) D. Shindo and K. Hiraga: High Resolution Electron Microscopy For Materials Science, (Springer, Tokyo, 1998).

5) J.C.H. Spence: Mater. Sci. Eng. R 26 (1999) 1-49.

6) S.J. Pennycook, S.D. Berger and R.J. Culbertson: J. Microscopy 144 (1986) 229-249.

7) S. Morishita, R. Ishikawa, Y. Kohno, H. Sawada, N. Shibata and Y. Ikuhara: Microscopy 67 (2018) 46-50.

8) N. Shibata, T. Seki, G. Sánchez-Santolino, S.D. Findlay, Y. Kohno, T. Matsumoto, R. Ishikawa and Y. Ikuhara: Nat. Commun. 8 (2017) 15631.

9) N. Shibata, Y. Kohno, A. Nakamura, S. Morishita, T. Seki, A. Kumamoto, H. Sawada, T. Matsumoto, S.D. Findlay and Y. Ikuhara: Nat. Commun. 10 (2019) 2308.

10) A. Tonomura: Electron Holography, 2nd ed., (Springer-Verlag, Berlin, 1999).

11) H. Lichte and M. Lehmann: Rep. Prog. Phys. 71 (2007) 016102.

12) E. Völkl, L.F. Allard and D.C. Joy: Introduction to Electron Holography, (Springer Science \& Business Media, Berlin, 1999).

13) M.R. McCartney and D.J. Smith: Annu. Rev. Mater. Res. 37 (2007) 729-767.

14) D. Shindo and T. Oikawa: Analytical Electron Microscopy for Materials Science, (Springer-Verlag, Tokyo, 2002).

15) D. Shindo: Mater. Trans. 44 (2003) 2025-2034.

16) K. Harada, T. Kohashi and M. Koguchi: Mater. Trans. 60 (2019) 2096 2102 .

17) Z. Akase and D. Shindo: Mater. Trans. 60 (2019) 2120-2124.

18) N. Kawamoto, H. Ono, Y. Terasaki, Y. Fujikawa, Y. Murakami and D. Shindo: Mater. Trans. 60 (2019) 2109-2113.

19) T. Sato, N. Tsukida, M. Higo, H. Magara, Z. Akase, D. Shindo and N. Ohno: Mater. Trans. 60 (2019) 2114-2119.

20) H. Nakajima, K. Shigematsu, Y. Horibe, S. Mori and Y. Murakami: Mater. Trans. 60 (2019) 2103-2108.

21) Y. Iwasaki, K. Harada, K. Shimada, M. Shirai and D. Shindo: Mater Trans. 61 (2020) 423-429.
22) Y. Murase, N. Miyauchi, A. Itakura and H. Katayama: Mater. Trans. 62 (2021) 41-47.

23) Y. Soejima, A. Heima, H. Akamine, T. Inamura and M. Nishida: Mater. Trans. 61 (2020) 2107-2114.

24) D. Shindo and Z. Akase: Mater. Sci. Eng. R 142 (2020) 100564.

25) K. Harada: Microscopy 70 (2021) 3-16.

26) Y. Cho, S. Lee and Y. Murakami: Microscopy 70 (2021) 17-23.

27) K. Yamamoto, S. Anada, T. Sato, N. Yoshimoto and T. Hirayama: Microscopy 70 (2021) 24-38.

28) T. Kawasaki, Y. Takahashi and T. Tanigaki: Microscopy 70 (2021) 3946.

29) M. Hÿtch and C. Gatel: Microscopy 70 (2021) 47-58.

30) S. Mori, H. Nakajima, A. Kotani and K. Harada: Microscopy 70 (2021) 59-68.

31) M. Mitome: Microscopy 70 (2021) 69-74.

32) M. Malac, S. Hettler, M. Hayashida, E. Kano, R.F. Egerton and M. Beleggia: Microscopy 70 (2021) 75-115.

33) J. Yamasaki: Microscopy 70 (2021) 116-130.

34) A.M. Blackburn and R.A. McLeod: Microscopy 70 (2021) 131-147.

35) T. Seki, Y. Ikuhara and N. Shibata: Microscopy 70 (2021) 148-160.

36) K. Harada: Appl. Phys. Lett. 100 (2012) 061901.

37) M. Uchida and A. Tonomura: Nature 464 (2010) 737-739.

38) J. Verbeeck, H. Tian and P. Schattschneider: Nature 467 (2010) 301304.

39) B.J. McMorran, A. Agrawal, I.M. Anderson, A.A. Herzing, H.J. Lezec, J.J. McClelland and J. Unguris: Science 331 (2011) 192-195.

40) K. Saitoh, Y. Hasegawa, N. Tanaka and M. Uchida: J. Electron Microsc. (Tokyo) 61 (2012) 171-177.

41) K.Y. Bliokh, M.R. Dennis and F. Nori: Phys. Rev. Lett. 107 (2011) 174802.

42) P. Schattschneider, M. Stöger-Pollach and J. Verbeeck: Phys. Rev. Lett. 109 (2012) 084801.

43) P. Schattschneider, S. Löffler, M. Stöger-Pollach and J. Verbeeck: Ultramicroscopy 136 (2014) 81-85.

44) B.J. McMorran, A. Agrawal, P.A. Ercius, V. Grillo, A.A. Herzing, T.R. Harvey, M. Linck and J.S. Pierce: Philos. Trans. R. Soc. London, Ser. A 375 (2017) 20150434.

45) K. Saitoh, Y. Hasegawa, K. Hirakawa, N. Tanaka and M. Uchida: Phys. Rev. Lett. 111 (2013) 074801.

46) V. Grillo, E. Karimi, G.C. Gazzadi, S. Frabboni, M.R. Dennis and R.W. Boyd: Phys. Rev. X 4 (2014) 011013.

47) V. Grillo, G. Carlo Gazzadi, E. Karimi, E. Mafakheri, R.W. Boyd and S. Frabboni: Appl. Phys. Lett. 104 (2014) 043109.

48) K. Yada, K. Shibata and T. Hibi: J. Electron Microsc. 22 (1973) 223230.

49) K.-J. Hanszen: J. Phys. D 19 (1986) 373-395.

50) M. Gajdardziska-Josifovska, M.R. McCartney, W.J. de Ruijter, D.J. Smith, J.K. Weiss and J.M. Zuo: Ultramicroscopy 50 (1993) 285-299.

51) A. Lubk, E. Javon, N. Cherkashin, S. Reboh, C. Gatel and M. Hÿtch: Ultramicroscopy 136 (2014) 42-49.

52) J.C.H. Spence and J.M. Zuo: Electron Microdiffraction, (Plenum Press, New York, 1992)

53) H. Kishi, Y. Okino, M. Honda, Y. Iguchi, M. Imaeda, Y. Takahashi, H. Ohsato and T. Okuda: Jpn. J. Appl. Phys. 36 (1997) 5954.

54) A.V. Polotai, T.-H. Jeong, G.-Y. Yang, E.C. Dickey, C.A. Randall, P. Pinceloup and A.S. Gurav: J. Electroceram. 18 (2007) 261-268.

55) C. Metzmacher and K. Albertsen: J. Am. Ceram. Soc. 84 (2001) 821826.

56) Y. Murakami, N. Kawamoto, D. Shindo, I. Ishikawa, S. Deguchi, K. Yamazaki, M. Inoue, Y. Kondo and K. Suganuma: Appl. Phys. Lett. 88 (2006) 223103.

57) N. Kawamoto, Y. Murakami, D. Shindo, H. Azehara and H. Tokumoto: Mater. Trans. 50 (2009) 1572-1575.

58) N. Kawamoto, Y. Kakefuda, I. Yamada, J. Yuan, K. Hasegawa, K. Kimoto, T. Hara, M. Mitome, Y. Bando, T. Mori and D. Golberg: Nano Energy 52 (2018) 323-328.

59) R. Kuramae, H. Ono, Y. Fujikawa, Y. Murakami and D. Shindo: Mater. Trans. 53 (2012) 696-699.

60) P.S. Bednyakov, B.I. Sturman, T. Sluka, A.K. Tagantsev and P.V. Yudin: npj Comput. Mater. 4 (2018) 65.

61) Y.S. Oh, X. Luo, F.-T. Huang, Y. Wang and S.-W. Cheong: Nat. Mater. 
14 (2015) 407-413.

62) J.A. Mundy et al:: Nat. Mater. 16 (2017) 622-627.

63) W. Wu, Y. Horibe, N. Lee, S.-W. Cheong and J.R. Guest: Phys. Rev. Lett. 108 (2012) 077203.

64) D. Meier, J. Seidel, A. Cano, K. Delaney, Y. Kumagai, M. Mostovoy, N.A. Spaldin, R. Ramesh and M. Fiebig: Nat. Mater. 11 (2012) 284288.

65) M.-G. Han, M.S.J. Marshall, L. Wu, M.A. Schofield, T. Aoki, R. Twesten, J. Hoffman, F.J. Walker, C.H. Ahn and Y. Zhu: Nat. Commun. 5 (2014) 4693

66) Z. Chen, X. Wang, S.P. Ringer and X. Liao: Phys. Rev. Lett. 117 (2016)
027601.

67) J.L. Hart, S. Liu, A.C. Lang, A. Hubert, A. Zukauskas, C. Canalias, R. Beanland, A.M. Rappe, M. Arredondo and M.L. Taheri: Phys. Rev. B 94 (2016) 174104.

68 D. Shindo, J. Jung Kim, K. Hyun Kim, W. Xia, N. Ohno, Y. Fujii, N. Terada and S. Ohno: J. Phys. Soc. Jpn. 78 (2009) 104802.

69) D. Shindo and Y. Murakami: Microscopy 60 (2011) S225-S237.

70) N. Ohno, H. Chiang, D.J. Mahad, G.J. Kidd, L. Liu, R.M. Ransohoff, Z.-H. Sheng, H. Komuro and B.D. Trapp: Proc. Natl. Acad. Sci. USA 111 (2014) 9953-9958. 